\title{
Effect of hearing aids on attention, memory, and auditory evoked potentials: a pragmatic, single-blinded, and randomized pilot clinical trial
}

\author{
Danilo Euclides Fernandes ${ }^{1}$, Gianna Mastroianni Kirsztajn ${ }^{1}$, and Katia de Almeida ${ }^{1}$ \\ ${ }^{1}$ Unifesp EPM
}

November 2, 2020

\begin{abstract}
Purpose: to compare, in a real-world scenario, the effects of different levels of technology on attention, memory, electrophysiological response, and self-perceived benefit of new users of hearing-aids in a developing country such as Brazil. Method: A pragmatic parallel-group, single-blinded, and randomized pilot clinical trial was conducted. Patients were divided into 3 groups according to hearing aid technology: (A) advanced technology; (B) basic technology; and (C) placebo. Participants were [?] 60 years old, had moderate sensorineural hearing loss, and had never been exposed to hearing aid before. Prior to data collection, patients were electronically randomized to receive unique identity numbers. Patient numbers were placed in opaque envelopes until the day of the first visit at which hearing aids were fitted with appropriate amplification settings. Attention, memory, and latency of auditory evoked potentials of patients were assessed while using the hearing aids at baseline and then after 12 weeks of use. The primary outcome was any improvement in scores on neuropsychological tests and/or shortening of latency in the auditory-evoked potentials. Results: A total of 22 individuals were assessed $(\mathrm{A}=8, \mathrm{~B}=6$, and $\mathrm{C}=8)$. Participants had a mean age of $80.4( \pm 6.1)$ years, were predominantly female $(63.63 \%)$, and were poorly educated (3.8 \pm 1.6 years). Comparison of groups AXC and of BXC revealed differences in NEUPSILIN scores (Brazilian instrument) for reverse counting ( $\mathrm{p}=0.002$, 95\% CI 5.9;20.55) and recognition ( $\mathrm{p}=0.013,95 \%$ CI -6.1;-0.88). No difference between groups A and B were found. Responses on the International Outcome Inventory for Hearing Aids (IOI-HA) differed for the benefit $(\mathrm{p}<0.001)$, satisfaction $(\mathrm{p}=0.007)$, participation restriction $(\mathrm{p}=0.012)$ and quality of life $(\mathrm{p}=0.037)$. Conclusion: The level of technology of the devices had no impact on the general satisfaction of new users of hearing-aids and appeared to have no differential effect on memory or attention after 12 weeks of use of the sound amplification products.
\end{abstract}

Effect of hearing aids on attention, memory, and auditory evoked potentials: a pragmatic, single-blinded, and randomized pilot clinical trial

Running title: Hearing aid technology and its effects

Danilo Euclides Fernandes ${ }^{\mathrm{I}}$, Gianna Mastroianni Kirsztajn ${ }^{\mathrm{II}}$, Katia de Almeida ${ }^{\mathrm{III}}$

I - MSci, MD-PhD Student at Universidade Federal de São Paulo (UNIFESP) - defernandes@unifesp.br ORCID: https://orcid.org/0000-0002-6324-1763

II - PhD, associated professor at Universidade Federal de São Paulo (UNIFESP), São Paulo - SP, Brazil gm.kirsztajn@unifesp.br - ORCID: https://orcid.org/0000-0003-1317-4109

II - PhD, professor at Faculdade de Ciências Médicas da Santa Casa de São Paulo - kalmeida@terra.com.br - ORCID: https://orcid.org/0000-0003-4247-6966

This study was conducted at Faculdade de Ciências Médicas da Santa Casa de São Paulo. 
Word count: 3026

The authors declare no conflicts of interest.

The authors declare no funding

The information within this manuscript was not submitted or presented before.

\section{Corresponding author information:}

Danilo Euclides Fernandes - Division of Nephrology

Botucatu St. 591, 15th floor, room 153, SP, Brazil

Postal code: 04023-900

Phone number: 5511 99983-2212

E-mail: defernandes@unifesp.br

\section{Abstract \\ Effect of hearing aids on attention, memory, and auditory evoked potentials: a pragmatic, single-blinded, and randomized pilot clinical trial}

Introduction: Population aging and its association with cognitive impacts of hearing loss is a growing concern. As hearing is restored and the auditory deprivation is reduced, the deleterious effects of hearing loss may be prevented or attenuated

Objective: to compare, in a real-world scenario, the effects of different levels of technology on attention, memory, electrophysiological response, and self-perceived benefit of new users of hearing-aids in a developing country such as Brazil.

Material and methods: A pragmatic parallel-group, single-blinded, and randomized pilot clinical trial was conducted. Patients were divided into 3 groups according to hearing aid technology: (A) advanced technology; (B) basic technology; and (C) placebo. Participants were [?] 60 years old, had moderate sensorineural hearing loss, and had never been exposed to hearing aid before. Prior to data collection, patients were electronically randomized to receive unique identity numbers. Patient numbers were placed in opaque envelopes until the day of the first visit at which hearing aids were fitted with appropriate amplification settings. Attention, memory, and latency of auditory evoked potentials of patients were assessed while using the hearing aids at baseline and then after 12 weeks of use. The primary outcome was any improvement in scores on neuropsychological tests and/or shortening of latency in the auditory-evoked potentials.

Results: A total of 22 individuals were assessed $(\mathrm{A}=8, \mathrm{~B}=6$, and $\mathrm{C}=8)$. Participants had a mean age of 80.4 (+-6.1) years, were predominantly female (63.63\%), and were poorly educated (3.8+-1.6 years). Comparison of groups A X C and of B X C revealed differences in NEUPSILIN scores (Brazilian instrument) for reverse counting ( $\mathrm{p}=0.002,95 \%$ CI 5.9;20.55) and recognition ( $\mathrm{p}=0.013,95 \%$ CI $-6.1 ;-0.88)$. No difference between groups $\mathrm{A}$ and $\mathrm{B}$ were found. Responses on the International Outcome Inventory for Hearing Aids (IOI-HA) differed for the benefit $(\mathrm{p}<0.001)$, satisfaction $(\mathrm{p}=0.007)$, participation restriction $(\mathrm{p}=0.012)$ and quality of life $(\mathrm{p}=0.037)$.

Conclusion: The level of technology of the devices had no impact on the general satisfaction of new users of hearing-aids and appeared to have no differential effect on memory or attention after 12 weeks of use of the sound amplification products.

Trial registration: UTN U1111-1250-9090; Brazilian Registry of Clinical Trials RBR-3Z98RP.

Funding: None

Keywords: Hearing Aids; Electrophysiology; Attention; Memory. 


\section{INTRODUCTION}

Population aging and its association with cognitive impacts of hearing loss [1-5] is a growing concern. As people age, cognitive dysfunction may manifest from mild to severe, including dementia itself [6], which represents a public health concern $[7,8]$ that might be considered a priority for public health $[8]$.

Hearing loss affects $23 \%$ of Americans who are 12 or older [9] and is considered the main modifiable factor capable of preventing cognitive decline in adult life [10]. As hearing is restored and the auditory deprivation is reduced, the deleterious effects of hearing loss can be prevented or attenuated, satisfaction with the use of hearing aids will increase, and auditory brain response will improve [11-20]. Also, despite the level of technology, both basic and premium hearing aids performs similarly among those with mild-to-moderate hearing loss $[21,22]$. Brazilian public health system, named SUS, freely provides hearing aids for those who have a medical prescription to use them.

Depending on the level of technology, hearing aids present some special sound processing strategies. The premium hearing aids typically include more complex, automatic and adaptative version of the features, as well as some features that are not available in the basic devices [23]. Among the features currently available are modern microphones, transposing frequencies, and cancelling feedback, besides amplifying the sound. Over the past years, both hardware (such as microphones) and software (directionality, noise reduction and such) have been evolving to a point in which microphones can adopt spatially dependent directivity pattern. Also, natural directivity that works as pinna-simulation directivity is an algorithm that seems to improve SNR. The premium hearing aids we used in this study accounts for all these features. More details on directionality, noise reduction, and sound processing algorithms can be found in some theorical books [24-26].

Although previous research have shown that hearing aids may impact on auditory brain response [20] and improve signal-to-noise ratio significantly [21,22], none of them describes pragmatically the hearing aids' effect on poor educated individuals. Thus, we aimed to compare, in a real-world scenario, the effects of different levels of technology on attention, memory, electrophysiological response, and self-perceived benefit of new users of hearing-aids in a developing country such as Brazil.

\section{MATERIALS AND METHODS}

\section{Design}

A pragmatic parallel-group, single-blinded, randomized pilot clinical trial was conducted (UTN U1111-12509090; Brazilian Registry of Clinical Trials RBR-3Z98RP) according to the recommendations of CONSORT, 2010 [27]. The study protocol was approved by the Research Ethics Committee of the Santa Casa de Sao Paulo (permit CAAE 50268715.1.0000.5479) and was devised in compliance with the principles of the Declaration of Helsinki. All participants signed the consent form.

\section{Participants}

Criteria for inclusion were individuals seen at the institution, aged [?] 60 years, with sensorineural, symmetric, and moderate hearing loss (air-conduction thresholds of 250-4000 Hz not exceeding $60 \mathrm{~dB}$ bilaterally) clinical indication of hearing aid use and naive to sound amplification products. Individuals who died or failed to attend the follow-up visits were excluded. Data collection was carried out between 2015 and 2016 .

We did not change the original methods we designed after this trial was started.

The flow diagram of enrollment, gain prescription, and algorithms activated in each group are depicted in Figure 1.

\section{Sample size calculation and randomization}

The sample size was not calculated because accurate data on the prevalence of cognitive decline in older patients with moderate hearing loss are lacking. 
The main author randomized the patients into three groups - A, B, and $\mathrm{C}$ - in a 1:1:1 ratio, using the Randomizer for Clinical Trials tool developed at the Medical University of Graz [28]. After random selection, the numbers and their respective groups were placed in opaque envelopes and sealed. Envelopes were opened at the time of selection of the hearing instruments to be fitted and the patients were blinded to their group allocation. Patient sequence, allocation, and treatment were carried out by the researchers.

\section{Setting and data collection}

All the data were collected by the main author, who have experience in fitting hearing aids. He conducted all the patients that were seen at the outpatient clinic of our institution (Santa Casa de Sao Paulo).

\section{Procedures and intervention}

During the first visit, a clinical history was taken. Also, the Montreal Cognitive Assessment (MoCA) was applied $[29,30]$ to guarantee no previous cognitive impairment (based on normative scores for hearing-impaired individuals [31]) and to exclude those individuals who could possibly introduce bias into the results of the proposed intervention. Hearing aids (receptor-in-the-ear, RIE) were then fitted according to the previously performed randomization. The acoustic gain prescription and the algorithms activated are described in Figure 1. After the first fitting, microphone probe measurements were made so as to ensure that both Groups $\mathrm{A}$ and B received at least $50 \%$ Speech Intelligibility Index (SII) in speech mapping for weak intensity sounds and at least $80 \%$ SII for medium-to-strong sounds. We activated the premium and basic features as shown in Figure 1. Checks were also carried out in Group C to ensure that the non-amplifying hearing-aid (placebo) did not exacerbate auditory deprivation of these control subjects. All participants were instructed on the use of the hearing aids and were given the contact of the researchers to remedy any problems arising during the use of the devices. After fitting the hearing aids, part of the Brief Neuropsychological Assessment Instrument was applied (NEUPSILIN) [32] to assess attention and memory abilities (for further details on this neuropsychological test, please read Fonseca et al., 2008 [33]). The patients we studied in this protocol did not pay for any device or health service as it happens in our health service which is financed by the Brazilian Public Health System (named SUS).

At the end of the consultation, cortical auditory responses were determined using a HEARLab(r) device according to the protocol recommended by Durante et al. [34] with speech stimuli $(/ \mathrm{m} /, / \mathrm{g} /$ and $/ \mathrm{t} /$ at intensities of 55, 65 and $75 \mathrm{~dB}$ ). Only latencies of P1 and N1 waves were employed. At the end of that visit, the next visit was scheduled (12 weeks later). During the period between the visits, the researchers contacted the patients every two weeks to ensure that hearing aids were being used properly.

Twelve weeks after the first visit, the participants returned to the service and were reassessed using the same NEUPSILIN tasks. Cortical auditory responses were measured again with the HEARLab(r) device according to the previously applied protocol. At the end of that visit, participants completed the International Outcome Inventory for Hearing Aids (IOI-HA), which investigated self-perceived usage, benefit, residual activity limitation, overall satisfaction, residual participation restriction, residual impact on others and quality of life change [35].

\section{Outcomes}

\section{Primary outcome}

The primary outcome was any of the following after 12 weeks of hearing aid use: 1) improving attention or memory scores on the NEUPSILIN; or 2) reducing latency of auditory evoked potentials, as measured by HEARLab(r) equipment. This variable was dichotomized into two categories: improved (1) and not improved (0).

Improvement in the NEUPSILIN scores was defined as a reduction (in seconds) in reverse counting time and/or higher total score on the tasks of digit sequence repetition, working memory, auditory word span, immediate and delayed recall, recognition, long-term memory evocation, and visual memory. Improvement in auditory evoked potentials was defined as a decrease in latency time $>1$ millisecond. 


\section{Secondary outcomes}

We considered the total score of IOI-HA as a secondary outcome, which meant the overall satisfaction with the hearing aids. This variable was analyzed as an ordinal one.

After the trial started, we included the total score of IOI-HA as a secondary outcome so that we could assess self-satisfaction with regards to hearing aid use at the end of the research.

\section{Statistical analysis}

Data were typed into Microsoft(r) Excel for Mac, version 16.35, and analyzed using IBM(r) SPSS Statistics software, version 26 for Mac. Data are shown as absolute and relative frequencies (nominal variables) and mean and standard deviation (continuous variables with non-normal distribution). Pearson's chi-square, ANOVA, Wilcoxon, and Mann-Whitney tests were employed to compare nominal and numerical variables and pre and post-use of hearing-aids, as needed, as well as Bonferroni correction (alpha $=0.05$; confidence interval $=95 \%)$. Also, we used the package $p w r$ in $\mathrm{R}(\mathrm{r})$ software to calculate the Pearson's chi-square power. As a pragmatic study, we applied the intention-to-treat method to analyze our data.

\section{RESULTS}

Figure 1 shows the flow diagram from enrollment to randomization, intervention, follow-up, and analysis.

All the patients were recruited from the waiting list of our service and those who met the inclusion criteria (elderly with mild-to-moderate sensorineural and naive to sound amplification) were invited to our outpatient clinic to receive their hearing aids. All the appointments (recruitment and follow-up) happened from a July 2015 to July 2016. The follow-up appointment was set in accordance with the protocol of our institution. Those who did not meet the 12 weeks of hearing aids use (after 3 months from the first appointment) were rescheduled to a next appointment as suggested in the current practice of hearing aid fitting of our institution. We stopped the trial because of the time we had to finish this study, which worked as a master's degree of the main author.

The final sample comprised 22 individuals who had a mean age of $80.4(+-6.1)$ years, were predominantly female $(63.63 \%)$, and had a mean education of $3.8(+-1.6)$ years. The participants randomized into the 3 groups $(\mathrm{A}=8, \mathrm{~B}=6$, and $\mathrm{C}=8)$ were analyzed by original assigned groups and did not differ for age, gender, education, cognitive screening scores on the MoCA (except language), hours of hearing aid use (informed and actual recorded), pure-tone audiometry (sensorineural, symmetric, and moderate hearing loss bilaterally), initial results on the NEUPSILIN tasks and auditory evoked potentials as shown in Table 1 and Supplementary Figure 1, which present the baseline characteristics of the three groups.

After fitting the hearing aids, in situ checks (probe measurements) revealed differences between individuals using sound amplification products (Groups A and B) and those using placebo (Group C) (Supplementary Table 1) as expected.

The analysis of IOI-HA responses showed differences in benefit $(\mathrm{p}<0.001)$, satisfaction $(\mathrm{p}=0.007)$, participation restriction $(\mathrm{p}=0.012)$ and quality of life $(\mathrm{p}=0.037)$. In addition, mean total scores on that inventory also differed across groups $(\mathrm{p}=0.032)$ (Table 1$)$.

As we conducted a pragmatic study, no subject was excluded, and we adopted an intention-to-treat approach in analysis.

The primary outcomes are given in Table 2 , showing statistically significant differences in reverse counting $(\mathrm{p}=0.03)$, auditory word span $(\mathrm{p}=0.03)$ and recognition $(\mathrm{p}=0.03)$ on the NEUPSILIN tasks and reduction in $\mathrm{N} 1$ wave latency for the $/ \mathrm{t} / \mathrm{phoneme}$ at a $55 \mathrm{~dB}$ intensity $(\mathrm{p}=0.01)$.

No potential harm was observed along with the follow-up of the patients. In the end of data collection, those who received the placebo intervention (group C) had their devices fitted according to the exact parameters we fitted the devices of both groups A and B. All the patients kept on ordinary follow-up at the outpatient clinic. 
The numerical results are summarized in Supplementary Tables 1 and 2.

Comparison of the numerical variables before and after 12 weeks of hearing aid use shows differences in scores on the NEUPSILIN for reverse counting ( $\mathrm{p}=0.006,95 \% \mathrm{CI} 2.2 ; 11.63)$, digit sequence repetition $(\mathrm{p}$ $=0.039,95 \% \mathrm{CI}-1.9 ;-0.05)$, delayed recall memory $(\mathrm{p}=0.036,95 \% \mathrm{CI}-1.2 ;-0.05)$, recognition $(\mathrm{p}=0.008$, $95 \% \mathrm{CI}-2.6 ;-0.45)$ and visual memory $(\mathrm{p}=0.008,95 \% \mathrm{CI}-0.9 ;-0.15)$ and in latency of potentials for the N1 wave for the $/ \mathrm{g} /$ phoneme at a $55 \mathrm{~dB}$ intensity $(\mathrm{p}=0.016,95 \% \mathrm{CI} 2.1 ; 18.59)$ (Supplementary Table 3).

Variables which differed before and after the intervention were submitted to a post hoc analysis, revealing differences between the sound amplification groups and the placebo group on NEUPSILIN scores for reverse counting $(\mathrm{p}=0.002,95 \% \mathrm{CI} 5.9 ; 20.55)$ and recognition $(\mathrm{p}=0.013,95 \% \mathrm{CI}-6.1 ;-0.88)$. This analysis found no differences among the groups for a reduction in N1 wave latency of the /g/ phoneme at $55 \mathrm{~dB}$. Likewise, no difference between groups A and B was found (Supplementary Table 3).

\section{DISCUSSION}

Our investigation presents data on comparison of different kinds of sound amplification technology (advanced and basic) for the first time described in a developing country. It also adds information on the short-term effect of hearing aids in attention and memory tasks and auditory evoked latencies, which should encourage further long-term studies.

The findings of the present study suggest the short-term beneficial effect of hearing-aid use in older adults who are naive to sound amplification devices, despite its level of technology [21,22].

With regard to the relationship between auditory deprivation and cognitive decline $[1-5,11-19]$, it is believed that the use of hearing aids represents a determinant for improving the quality of life among hearing-impaired elderly [36]. To date, we have no instrument that can directly assess cognition as a whole, so we had to rely on attention and memory skills, as well as auditory evoked potentials latency to hypothesize so.

The educational level of our sample, comprising older patients who depend on the public health service, is representative of the majority of the population of the developing countries such as ours. Consequently, the findings of this study only represent those countries with a similar sociodemographic profile. Poor education is considered a potentially modifiable risk factor for dementia [10], particularly in countries such as Brazil [37]. Therefore, strategies for mitigating these risks are vital in these countries, which plays an effort to minimize cognitive impairment. Given Brazil's rapidly aging population, auditory deprivation may constitute a factor that limits the quality of life of the elderly. Auditory deprivation is held as the main modifiable factor in adult life capable of preventing cognitive decline [10]. The role of audiologists and ENT specialists is thus to minimize hearing deprivation by diagnosing hearing loss as soon as they can.

The hearing aids we used in this study reflect the different levels of technology consistent with the devices available at the time of this clinical trial. Group A used devices of premium technology, including features of automatic asymmetric directionality, environmental acoustic gain optimizer (up to $6 \mathrm{~dB}$ gain for less noisy environments), and noise reduction by environment (noise reduction of up to $10 \mathrm{~dB}$ in noisy environments). Group B (basic technology) used hearing aids with fixed symmetric directionality (omnidirectional OR directional simultaneously), with no gain optimizer by environment, and offering maximum noise reduction of $3 \mathrm{~dB}$. Both Groups A and B used default manufacturer settings (GN group) for wind-noise reduction, microphone feedback cancelation, and expansion. Group C (placebo) patients, after using their hearing aids for 12 weeks with no sound amplification, had their devices programmed according to the same protocol applied for fitting groups A and B. All the electroacoustic characteristics we mentioned here are easily found in the technical specifications of the hearing aids from the GN group (ReSound).

As shown by a previous studies [21,22,38], our results failed to show any difference between premium and basic hearing aids, which suggest that amplification plays the major role in restoring hearing abilities. It is believed that sound signal processing algorithms may reduce listening effort [16-19], but robust data supporting these theories are still lacking. 
Although we chose a neuropsychological test that was not sensitive enough for detecting discrete improvements in memory and attention, the NEUPSILIN was elected for the present study because it is validated for its use in older Brazilian patients and thus could be applied in our sample. One of the tests which differed statistically between pre and post-intervention was the word recognition task. On this task, the subject hears the spoken words aloud by the examiner and must decide whether these are part of the list of words they heard earlier in the test. In this assessment, the patient must deal with phonemic distractors, thus the sound amplification itself facilitates the patient's performance at it. A comparison of the 3 groups revealed that this difference occurred only between Groups B and C, pointing to a possible sample size effect.

The definition used by Moore et al. [39] was originally proposed by Neisser in 1967 and states that cognition is an individual's ability to transform sensorial input into meaning. Hearing aids allow the individuals to perceive sounds again, but the reassignment of meaning to these sound stimuli is not immediate and might not take place spontaneously. In this respect, auditory training and/or speech-hearing therapy may help hearing impaired people to derive maximum benefit from sound amplification.

We believe the reassessment with auditory evoked potentials may have been carried out too early, which prevented the plasticity of the patients' auditory system to happen. We also speculate if latency could be rapidly affected by amplification as waves amplitude were [20].

The differences we observed on the IOI-HA were more likely associated with sound amplification, regardless of the technology level. This suggests that for new users, a well-fitted hearing aid may be the main factor that guides satisfaction.

\section{Limitations}

The main limitations of this study, besides sample size and blinding type, are the limited sensitivity of the methods we used for assessing patients pre and post-intervention and the short period of use of hearing aids use. Given the dearth of studies on this topic, the assessment methods chosen in this study are not deemed a gold standard for cognitive assessment, despite the adoption of intra-subject analyses (comparing the individual against themselves after the intervention over the time). The period of use of hearing aids up to the reassessment was very short, precluding the detection of the intervention effects by the assessments we performed in this study. It is worth to mention this is a pragmatic pilot clinical trial. It may encourage researches worldwide to conduct other studies that help audiologists and ENTs to better understand how the hearing aids affect their patients in both short and long-term.

\section{CONCLUSION}

In this study, differences in technology had no impact on general customer satisfaction nor in the cognitive tasks such as memory and attention after 12 weeks of using hearing aids among individuals who were naive to sound amplification

The significant differences we observed in this study seem to be related to avoiding sound deprivation (through hearing aids use) for both objective and neuropsychological tests and general customer satisfaction.

\section{CLINICAL TRIAL PROTOCOL REGISTRATION}

This protocol was registered at Brazilian Registry of Clinical Trials (RBR-3Z98RP) and it can be fully accessed at http://www.ensaiosclinicos.gov.br/rg/RBR-3z98rp/.

FUNDING: None

\section{ACKNOWLEDGEMENTS}

The authors extend their thanks to the GN group for the advanced technology hearing aids made available by means of the Outpatient Procedure Authorization at the rate prevailing in the National Health System (SUS) at the time of data collection.

\section{CONTRIBUTION OF AUTHORS}


Danilo Euclides Fernandes: data collection, analysis, and discussion, manuscript preparation and approval of the final version of the manuscript.

Gianna Mastroianni Kirsztajn: data analysis and discussion, manuscript preparation and approval of the final version of the manuscript.

Katia de Almeida: Project coordination, data analysis and discussion, manuscript preparation and approval of the final version of the manuscript.

References

1. Uhlmann RF, Larson EB, Rees TS, Koepsell TD, Duckert LG. Relationship of hearing impairment to dementia and cognitive dysfunction in older adults. JAMA. 1989 ; 261(13):1916-1919.

2. Lin FR. Hearing loss and cognition among older adults in the United States. J Gerontol A Biol Sci Med Sci. $2011 ; 66(10): 1131-1136$.

3. Lin FR, Yaffe K, Xia J, et al. Hearing loss and cognitive decline in older adults. JAMA Intern Med. $2013 ; 173(4): 293-299$.

4. Lin FR, Albert M. Hearing loss and dementia - who is listening? Aging Ment Health. 2014 ; 18(6):671673.

5. Peelle JE, Troiani V, Grossman M, Wingfield A. Hearing loss in older adults affects neural systems supporting speech comprehension. J Neurosci Off J Soc Neurosci. 2011 ; 31(35):12638-12643.

6. Ataollahi Eshkoor S, Mun CY, Ng CK, Hamid TA. Mild cognitive impairment and its management in older people. Clin Interv Aging.2015; :687.

7. Alzheimer's Association. 2015 Alzheimer's disease facts and figures. Alzheimers Dement. 2015 ; 11(3):332-384.

8. Frankish H, Horton R. Prevention and management of dementia: a priority for public health. The Lancet. 2017 ; 390(10113):2614-2615.

9. Goman AM, Lin FR. Prevalence of Hearing Loss by Severity in the United States. Am J Public Health. 2016 ; 106(10):1820-1822.

10. Livingston G, Sommerlad A, Orgeta V, et al. Dementia prevention, intervention, and care. The Lancet. $2017 ; 390(10113): 2673-2734$.

11. Gatehouse S, Naylor G, Elberling C. Benefits from hearing aids in relation to the interaction between the user and the environment. Int J Audiol. 2003 ; 42 Suppl 1:S77-85.

12. Foo C, Rudner M, Ronnberg J, Lunner T. Recognition of speech in noise with new hearing instrument compression release settings requires explicit cognitive storage and processing capacity. J Am Acad Audiol.2007 ; 18(7):618-631.

13. Souza PE, Arehart KH, Kates JM, Croghan NBH, Gehani N. Exploring the limits of frequency lowering. J Speech Lang Hear Res JSLHR.2013 ; 56(5):1349-1363.

14. Ellis RJ, Munro KJ. Does cognitive function predict frequency compressed speech recognition in listeners with normal hearing and normal cognition? Int J Audiol. 2013 ; 52(1):14-22.

15. Ng EHN, Rudner M, Lunner T, Pedersen MS, Ronnberg J. Effects of noise and working memory capacity on memory processing of speech for hearing-aid users. Int J Audiol. 2013 ; 52(7):433-441.

16. Neher T, Grimm G, Hohmann V, Kollmeier B. Do hearing loss and cognitive function modulate benefit from different binaural noise-reduction settings? Ear Hear. 2014 ; 35(3):e52-62. 
17. Desjardins JL, Doherty KA. The effect of hearing aid noise reduction on listening effort in hearingimpaired adults. Ear Hear. $2014 ; 35(6): 600-610$.

18. Neher T. Relating hearing loss and executive functions to hearing aid users' preference for, and speech recognition with, different combinations of binaural noise reduction and microphone directionality. Front Neurosci. $2014 ; 8: 391$.

19. Ng EHN, Rudner M, Lunner T, Ronnberg J. Noise reduction improves memory for target language speech in competing native but not foreign language speech. Ear Hear. 2015 ; 36(1):82-91.

20. Seol HY, Park S, Ji YS, Hong SH, Moon IJ. Impact of hearing aid noise reduction algorithms on the speech-evoked auditory brainstem response. Sci Rep. 2020 ; 10(1):10773.

21. Johnson JA, Xu J, Cox RM. Impact of Hearing Aid Technology on Outcomes in Daily Life II: Speech Understanding and Listening Effort. Ear Hear. 2016 ; 37(5):529-540.

22. Wu Y-H, Stangl E, Chipara O, Hasan SS, DeVries S, Oleson J. Efficacy and Effectiveness of Advanced Hearing Aid Directional and Noise Reduction Technologies for Older Adults With Mild to Moderate Hearing Loss: Ear Hear. 2019 ; 40(4):805-822.

23. Cox RM, Johnson JA, Xu J. Impact of Hearing Aid Technology on Outcomes in Daily Life I: The Patients' Perspective. Ear Hear.2016 ; 37(4):e224-e237.

24. Popelka GR. Hearing aids: basic and applied. New York, NY: Springer Science+Business Media; 2016.

25. Dillon H. Hearing aids. 2. ed. Sydney: Boomerang Press [u.a.]; 2012.

26. Metz MJ, editor. Sandlin's textbook of hearing aid amplification: technical and clinical considerations. Third edition. San Diego, CA: Plural Publishing; 2014.

27. Moher D, Hopewell S, Schulz KF, et al. CONSORT 2010 explanation and elaboration: updated guidelines for reporting parallel group randomised trials. Int J Surg Lond Engl. $2012 ; 10(1): 28-55$.

28. Medical University of Graz. Randomizer [Internet]. [cited 2015 Jan 14]. Available from: http://www.randomizer.at/

29. Nasreddine ZS, Phillips NA, Bedirian V, et al. The Montreal Cognitive Assessment, MoCA: a brief screening tool for mild cognitive impairment. J Am Geriatr Soc. 2005 ; 53(4):695-699.

30. Apolinario D, Dos Santos MF, Sassaki E, et al. Normative data for the Montreal Cognitive Assessment (MoCA) and the Memory Index Score (MoCA-MIS) in Brazil: Adjusting the nonlinear effects of education with fractional polynomials. Int J Geriatr Psychiatry. 2018 ; 33(7):893-899.

31. Dupuis K, Pichora-Fuller MK, Chasteen AL, Marchuk V, Singh G, Smith SL. Effects of hearing and vision impairments on the Montreal Cognitive Assessment. Neuropsychol Dev Cogn B Aging Neuropsychol Cogn.2015 ; 22(4):413-437.

32. Fonseca R, Salles J, Parente M. NEUPSILIN - Instrumento de avaliacao neuropsicologica breve (Brief Neuropsychological Assessment Instrument). Sao Paulo: Vetor; 2009.

33. Fonseca RP, Salles JF de, Parente MA de MP. Development and content validity of the Brazilian Brief Neuropsychological Assessment Battery Neupsilin. Psychol Amp Neurosci. Pontificia Universidade Catolica do Rio de Janeiro; 2008 ; 1(1):55-62.

34. Durante AS, Wieselberg MB, Carvalho S, et al. Cortical Auditory Evoked Potential: evaluation of speech detection in adult hearing aid users. CoDAS. Sociedade Brasileira de Fonoaudiologia; 2014 ; 26(5):367-373.

35. Cox RM, Alexander GC, Beyer CM. Norms for the international outcome inventory for hearing aids. J Am Acad Audiol. 2003 ; 14(8):403-413. 
36. Ribeiro UASL, Souza VC, Lemos SMA. Quality of life and social determinants in individual hearing AIDS users. CoDAS. 2019 ; 31(2):e20170287.

37. Mukadam N, Sommerlad A, Huntley J, Livingston G. Population attributable fractions for risk factors for dementia in low-income and middle-income countries: an analysis using cross-sectional survey data. Lancet Glob Health. 2019 ; 7(5):e596-e603.

38. Cho YS, Park SY, Seol HY, et al. Clinical Performance Evaluation of a Personal Sound Amplification Product vs a Basic Hearing Aid and a Premium Hearing Aid. JAMA Otolaryngol Neck Surg. American Medical Association; 2019 ; 145(6):516-522.

39. Moore DR, Edmondson-Jones M, Dawes P, et al. Relation between speech-in-noise threshold, hearing loss and cognition from 40-69 years of age. PloS One. $2014 ; 9(9)$ :e107720.

FIGURES

Figure 1. Flow diagram of enrollment process according to CONSORT, 2010 [27].

Abbreviations: n, sample.

Table 1. Demographic data and baseline characteristics of Groups A (advanced technology), B (basic technology) and C (placebo) for MoCA screening, NEUPSILIN scores, Speech Intelligibility Index (SII) and IOI-HA.

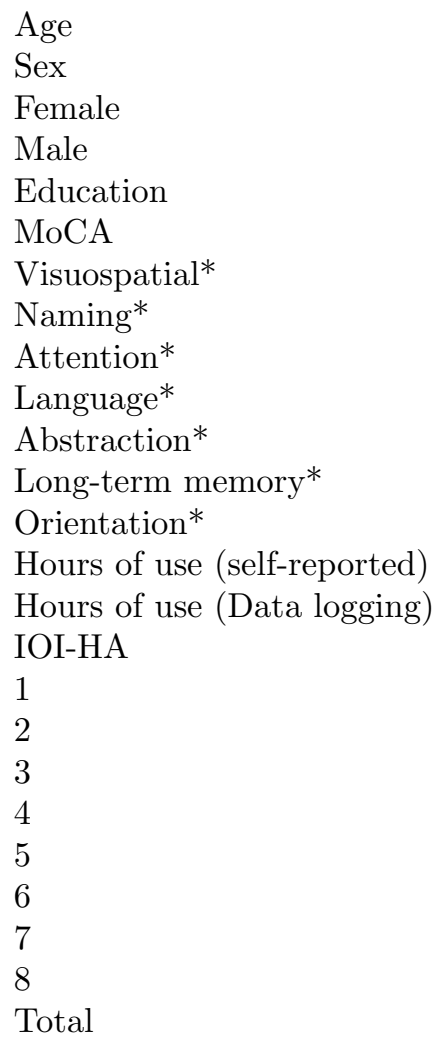

a One-way ANOVA b Pearson's chi-squared *The higher the score, the better the result is.

Abbreviations: dB, decibel; IOI-HA, Inventory Outcome Index of Hearing Aids; MoCA, Montreal Cognitive Assessment; 
Table 2. Absolute and relative frequencies for primary outcomes by group.

NEUPSILIN, n (\%)

Reverse counting

Digit sequence repetition

Working memory

Auditory digit span

Short-term memory

Long-term memory

Recognition

Long-term memory

Visual memory

Evoked potentials, $\mathrm{n}(5)$

$55 \mathrm{~dB}$

$\mathrm{P} 1 / \mathrm{m} /$

$\mathrm{N} 1 / \mathrm{m} /$

$\mathrm{P} 1 / \mathrm{t} /$

N1/t/

P1 /g/

N1 /g/

$65 \mathrm{~dB}$

$\mathrm{P} 1 / \mathrm{m} /$

$\mathrm{N} 1 / \mathrm{m} /$

$\mathrm{P} 1 / \mathrm{t} /$

N1/t/

$\mathrm{P} 1 / \mathrm{g} /$

N1 /g/

$75 \mathrm{~dB}$

$\mathrm{P} 1 / \mathrm{m} /$

$\mathrm{N} 1 / \mathrm{m} /$

$\mathrm{P} 1 / \mathrm{t} /$

N1 / t/

$\mathrm{P} 1 / \mathrm{g} /$

N1 /g/

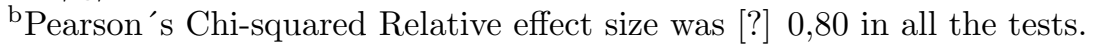

Abbreviations: dB, decibel; IOI-HA, Inventory Outcome Index of Hearing Aids; N1, N1 wave; NEUPSILIN, Brief Neurop

\section{SUPPLEMENTARY MATERIALS}

Supplementary Table 1. Demographic data and baseline characteristics of Groups A (advanced technology), B (basic tes

Age

Sex

Female

Male

Education

$\mathrm{MoCA}$

Visuospatial*

Naming*

Attention* 
Supplementary Table 1. Demographic data and baseline characteristics of Groups A (advanced technology), B (basic te

Language*

Abstraction*

Long-term memory*

Orientation*

Hours of use (self-reported)

Hours of use (Data logging)

Audiometry, dB (SD)

Right ear

$250 \mathrm{~Hz}$

$500 \mathrm{~Hz}$

$1000 \mathrm{~Hz}$

$2000 \mathrm{~Hz}$

$3000 \mathrm{~Hz}$

$4000 \mathrm{~Hz}$

$6000 \mathrm{~Hz}$

$8000 \mathrm{~Hz}$

SRI

Left ear

$250 \mathrm{~Hz}$

$500 \mathrm{~Hz}$

$1000 \mathrm{~Hz}$

$2000 \mathrm{~Hz}$

$3000 \mathrm{~Hz}$

$4000 \mathrm{~Hz}$

$6000 \mathrm{~Hz}$

$8000 \mathrm{~Hz}$

SRI

NEUPSILIN

Before

Reverse counting, s, (SD)**

Digit sequence repetition, right answers $(\mathrm{SD})^{*}$

Working memory, right answers (SD)*

Auditory digit span, right answers (SD)*

Short-term memory, right answers (SD)*

Long-term memory, right answers (SD)*

Recognition, right answers (SD)*

Long-term memory, right answers (SD)*

Visual memory, right answers (SD)*

Evoked potential

Before

$55 \mathrm{~dB}$

$\mathrm{P} 1 / \mathrm{m} /$

$\mathrm{N} 1 / \mathrm{m} /$

$\mathrm{P} 1 / \mathrm{t} /$

$\mathrm{N} 1 / \mathrm{t} /$

$\mathrm{P} 1 / \mathrm{g} /$

$\mathrm{N} 1 / \mathrm{g} /$

$65 \mathrm{~dB}$

$\mathrm{P} 1 / \mathrm{m} /$

$\mathrm{N} 1 / \mathrm{m} /$ 
Supplementary Table 1. Demographic data and baseline characteristics of Groups A (advanced technology), B (basic te

$\mathrm{P} 1 / \mathrm{t} /$

N1 / t /

P1 /g/

N1 /g/

$75 \mathrm{~dB}$

$\mathrm{P} 1 / \mathrm{m} /$

$\mathrm{N} 1 / \mathrm{m} /$

$\mathrm{P} 1 / \mathrm{t} /$

N1 / t/

P1 /g/

N1 /g/

SII

Right ear

$55 \mathrm{~dB}$

$65 \mathrm{~dB}$

$75 \mathrm{~dB}$

Left ear

$55 \mathrm{~dB}$

$65 \mathrm{~dB}$

$75 \mathrm{~dB}$

a One-way ANOVA with power [?] 0,50. ${ }^{*}$ The higher the score, the better the result is. ${ }^{*}$ The higher the score, the worse Abbreviations: dB, decibel; IOI-HA, Inventory Outcome Index of Hearing Aids; SRI, Speech Recognition Index; MoCA,

Supplementary Table 2. Comparison pre and post-intervention involving hearing aids use.

\section{NEUPSILIN}

Reverse counting, s (SD)**

Digit sequence repetition, right answers $(\mathrm{SD})^{*}$

Working memory, right answers (SD)*

Auditory digit span, right answers (SD)*

Short-term memory, right answers $(\mathrm{SD})^{*}$

Long-term memory, right answers (SD)*

Recognition, right answers (SD)*

Long-term memory, right answers (SD)*

Visual memory, right answers (SD)*

Evoked potential, ms (SD)

$55 \mathrm{~dB}$

$\mathrm{P} 1 / \mathrm{m} /$ 
Supplementary Table 2. Comparison pre and post-intervention involving hearing aids use.

$\mathrm{N} 1$ /m/

$\mathrm{P} 1 / \mathrm{t} /$

$\mathrm{N} 1 / \mathrm{t} /$

P1 /g/

N1 /g/

$65 \mathrm{~dB}$

$\mathrm{P} 1 / \mathrm{m} /$

$\mathrm{N} 1 / \mathrm{m} /$

$\mathrm{P} 1 / \mathrm{t} /$

$\mathrm{N} 1 / \mathrm{t} /$

$\mathrm{P} 1 / \mathrm{g} /$

N1 /g/

$75 \mathrm{~dB}$

$\mathrm{P} 1 / \mathrm{m} /$

$\mathrm{N} 1 / \mathrm{m} /$

$\mathrm{P} 1 / \mathrm{t} /$

$\mathrm{N} 1 / \mathrm{t} /$

$\mathrm{P} 1 / \mathrm{g} /$

N1 /g/

c Wilcoxon test (pre and post-intervention). ${ }^{*}$ The higher the score, the better the result it. ${ }^{* *}$ The higher the score, the wo

Supplementary Table 3. Initial (i) and final (f) assessments and pairwise comparisons (A X C. B X C and A X B).

NEUPSILIN

Reverse counting, $\mathrm{s},(\mathrm{SD})^{* *}$

Digit sequence repetition, right answers $(\mathrm{SD})^{*}$ 
Long-term memory, right answers (SD)*

Recognition, right answers (SD)*

Visual memory, right answers (SD)*

Evocated potentials, ms (SD)

$55 \mathrm{~dB}$

N1 /g/

c Wilcoxon text (pre and post intervention). d Mann-Whitney test ${ }^{*}$ The higher the score, the better the result it. $* *$ The $\mathrm{h}$ Abbreviations: A, advanced technology; B, basic technology; C, placebo; CI, confidence interval; NEUPSILIN, Brief Neur 